\title{
BUCKLED BEAM LINEAR OUTPUT CAPACITIVE STRAIN SENSOR
}

\author{
J. Guo, H. Kuo, D. J. Young, W. H. Ko \\ EECS Department, Case Western Reserve University \\ Cleveland, $\mathrm{OH}, 44106$
}

\begin{abstract}
A capacitive strain sensor employing a mechanical amplification scheme has been designed, analyzed, fabricated, and tested. The mechanical amplifier based on the bending of a buckled beam provides an increased device sensitivity, thus attractive on reducing the sensitivity requirement and power dissipation in the interface circuits. An analytical model was developed to predict the mechanical gain of the structure, which was confirmed by Finite-Element-Analysis (FEA), with an error of less than $1.5 \%$ of the measurement results. The fabricated device achieved a sensitivity of 282 atto-farads/microstrain $(\mathrm{aF} / \mu \varepsilon)$. The mechanical thermal noise analysis shows that a sensitivity of 0.1 microstrains $(\mu \varepsilon)$ with a dynamic range of $10,000(80 \mathrm{~dB})$ over 10 $\mathrm{kHz}$ bandwidth can be achieved combining this design with a matching interface circuit.
\end{abstract}

\section{INTRODUCTION}

High-performance strain sensors having large dynamic range and bandwidth are highly desirable in applications such as aerospace, biomedical and power industries. Traditional metal foil strain sensors dominate current market because of their low cost and easiness to handle. However, foil strain sensors suffer from low sensitivity with gauge factors typically around 2 and high power consumption [1]. Silicon piezoresistive strain sensors employing the piezoresistivity effect, which is a phenomenon that a material changes its resistance with stress, can achieve gauge factors typically from 30 to 120 . However, the piezoresistive strain sensors suffer from high temperature instability, initial drift, and large resistive thermal noise and power dissipation. Capacitive strain sensors can overcome the above drawbacks and achieve a large dynamic range and bandwidth with a reduced power consumption. This is particularly attractive in biomedical applications where low-power consumption devices are critical. However, the minimum detectable capacitance change of most MEMS capacitive devices is typically on the order of tens of attofarads $(\mathrm{aF})$; hence, high-sensitivity integrated or nearby interface circuits, and sophisticated packaging schemes are required to maintain an overall system performance, which typically results in high power consumption and cost. Therefore, a capacitive strain sensor with a mechanical amplification is highly attractive as it improves the sensor sensitivity, thus reducing the noise requirement and power dissipation for interface circuits.

Researches were reported on measuring residual stresses utilizing an mechanical amplification scheme achieving a strain resolution of 10 microstrain [2,3]. This paper presents an innovative capacitive strain sensor structure that combines the mechanical amplification of a buckled beam and the comb structure to achieve a high sensitivity below 0.1 microstrain $(\mu \varepsilon)$ and large linear dynamic range up to $80 \mathrm{db}$. An analytical model has been developed to predict the mechanical gain of the structure, which has been verified by ANSYS FEA.

\section{DESIGN CONCEPTS}

The schematic diagram of the buckled beam mechanical amplification scheme is shown in Figure 1. The structure consists of two buckled beams with a small buckling angle, $\alpha$, and one sensing beam at center. When a strain, $\varepsilon$, is applied, it causes a lateral displacement, $\Delta x=L_{g} \varepsilon$. For a small buckling angle, the center deflection of the sensing beam, $\Delta w$, is larger than $\Delta x$, thus resulting in a mechanical amplification gain, $A_{\text {mech }}$. A linear differential capacitive output can be obtained by designing comb drive fingers at the center sensing beam, as shown in Figure 2. For a compressive strain, the top and bottom beams will move up and the center beam will move down, thus resulting in a differential capacitance change.

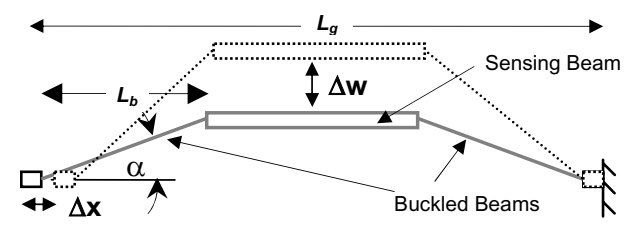

Figure 1. Principle of the buckled beam amplification scheme.

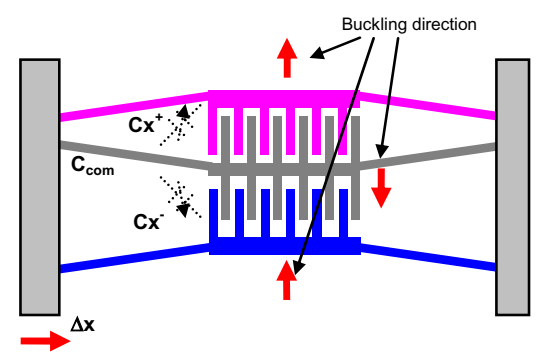

Figure 2. A buckled beam strain sensor with differential output.

\section{MODELING}

A. Beam modeling

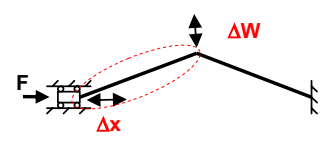

(a)

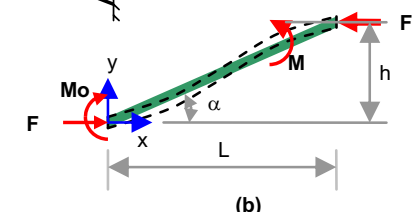

Figure 3. (a) The simplified structure. (b) Forces acting on the beam. 
An analytical analysis based on the simple beam theory was developed to predict the mechanical gain of the buckled beam. The sensor structure can be simplified as a simple beam structure constrained as shown in Figure 3.a with one end fixed and the other end guided along the $\mathrm{x}$-axis. The forces acting on the bent beam are shown in Figure 3.b, where $F$ is the equivalent force applied by the external strain, $M o$ is moment from the guild end, $M$ is the moment in the beam at an arbitrary location $(\mathrm{x}, \mathrm{y})$, and the governing equation is given by [5],

$$
E I \frac{d^{2} y}{d x^{2}}=M_{o}=M-F y,
$$

where $E$ and $I$ are the Young's modulus and moment of inertia of the beam, respectively.

The boundary conditions are given by,

$$
\left.y\right|_{x=0}=0,\left.\frac{d y}{d x}\right|_{x=0}=\tan \alpha, \text { and }\left.\frac{d y}{d x}\right|_{x=L}=\tan \alpha .
$$

By solving the above equations, and assuming that the changes of angle, $\alpha$, can be neglected, the center deflection, $\Delta w$, can be expressed by [3],

$$
\begin{array}{lr}
\Delta w=2 \frac{\tan \alpha}{k} \tan (k L / 2)-h, & \text { (for compression) (3a) } \\
\Delta w=2 \frac{\tan \alpha}{k} \tanh (k L / 2)-h, & \text { (for tension) (3b) }
\end{array}
$$

where $k=\sqrt{F / E I}$.

The end displacement of the buckled beam can be calculated by,

$$
\begin{aligned}
& \Delta x=\int_{0}^{L} \frac{1}{2}\left(\frac{d y}{d x}\right)^{2} d x-L \frac{1}{2} \tan ^{2} \alpha, \quad \text { (for compression) (4a) } \\
& \Delta x=\int_{0}^{L} \frac{1}{2}\left(\frac{d y}{d x}\right)^{2} d x-L \frac{1}{2} \tanh ^{2} \alpha, \quad \text { (for tension) (4b) }
\end{aligned}
$$

Therefore, for a small buckling angle and small lateral displacement, the mechanical gain of the buckled beam can be solved as,

$$
A_{\text {mech }}=\frac{\Delta w}{\Delta x} \cong \frac{1}{\tan \alpha} .
$$

An FEA analysis using ANSYS has been performed to confirm the above analytical results. Table 1 shows the comparison between the results obtained analytically using Equation (5) and by FEA by apply a small strain such as $100 \mu \varepsilon$. An error less than $1 \%$ was observed, indicating a high accuracy of the modeling.

Table 1. Comparison of the mechanical gain obtained analytically and by FEA for a buckled bema with length of $300 \mu \mathrm{m}$ and width of $3 \mu \mathrm{m}$.

\begin{tabular}{|c|c|c|c|c|c|}
\hline Beam height, $\mathrm{h}(\mathrm{um})$ & 30 & 40 & 50 & 60 & 70 \\
\hline $\tan (\alpha)$ & 0.100 & 0.133 & 0.167 & 0.200 & 0.233 \\
\hline Calculated Gain & 10.000 & 7.500 & 6.000 & 5.000 & 4.286 \\
\hline ANSYS result & 9.900 & 7.450 & 5.970 & 4.980 & 4.270 \\
\hline Error $(\%)$ & 1.000 & 0.667 & 0.500 & 0.400 & 0.367 \\
\hline
\end{tabular}

\section{B. Sensor modeling}

Based on the results of the beam modeling, the sensor sensitivity, which is defined as the capacitance change per unit applied strain, can be expressed as,

$$
S \equiv \frac{\Delta C}{\Delta \text { Strain }}=\frac{4 N A_{\text {mech }} t \varepsilon_{e}}{g},
$$

where $N$ is the number of fingers on each sensing beam, $A_{\text {mech }}$ is the mechanical gain, $t$ is the thickness of the beam, $\varepsilon_{e}$ is the dielectric permittivity, and $g$ is the gap between fingers.

An FEA analysis of a full device structure employing a coupled-field analysis method has been performed to verify the above analytical results. Figure 4 shows the capacitance change characteristics obtained by using Equation (6) and FEA. A maximum $11 \%$ error for compressive strain and a $5 \%$ error for tensile strain at range of $1000 \mu \varepsilon$ are observed. These errors are introduced by the change of the beam buckling angle, $\alpha$, as the applied strain increases and can be improved by optimizing the structure parameters. A nonlinearity analysis of the FEA curve shows a nonlinearity of $1.49 \% \mathrm{FS}$ for the range of 0 to $1000 \mu \varepsilon$ tensile strain, which agrees with measurement results.

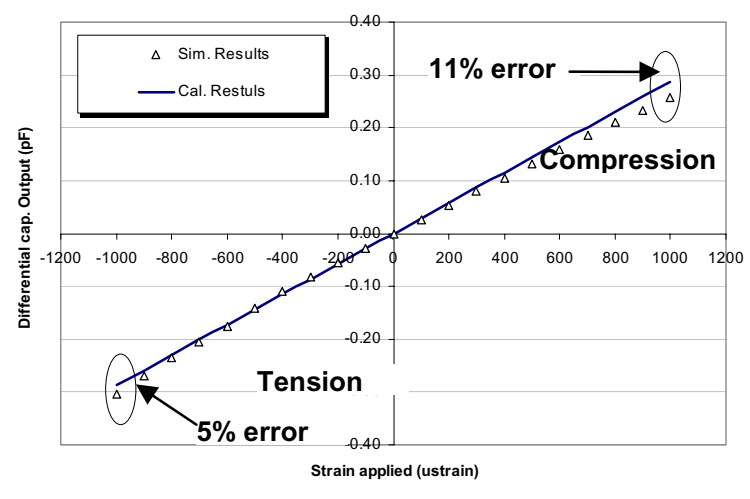

Figure 4. Simulation results about capacitance change as a function of load strain.

\section{Device resolution analysis}

The fundamental sensing resolution is determined by the device noise such as the resistive thermal noise introduced by the device series resistances and the mechanical thermal noise of the structure (Brownian motion) [4]. The mechanical thermal noise is the dominate part as the series resistance is typically on the order of $100 \mathrm{ohms}$ or less. The mechanical thermal noise is introduced by the air damping of a structure and for a second-order mechanical damping system such as the designed strain sensor, the noise is basically determined by the structure mechanical parameters such as resonant frequency $\left(\omega_{0}\right)$, damping factor (or quality factor Q) and mass (m). The input strain referred equivalent thermal noise is given by,

$$
\varepsilon_{n}=K_{n} \sqrt{\frac{4 K_{B} T}{\omega_{o}{ }^{3} m Q}} \cdot \sqrt{B W} .
$$

where $\varepsilon_{\mathrm{n}}$ is input strain referred mechanical thermal noise; $K_{B}$ is the Boltzman constant; $T$ is the absolute temperature with unit of Kelvin; $\omega_{o}$ is the structure resonant frequency; $m$ is the mass; $Q$ is the quality factor; $B W$ is the required signal bandwidth; $K_{\mathrm{n}}$ is the conversion factor between the noise displacement and the inputreferred strain, which is a function of mechanical gain and gauge length, and equals to 200 according to the sensor dimensions given in Table 2.

Table 3 lists the mechanical parameters of a designed device; the calculated input strain referred mechanical thermal noise is less than $0.003 \mu \varepsilon$ for a bandwidth of $10 \mathrm{kHz}$. A low noise charge amplifier has been designed to interface with the sensor to achieve a sensitivity of $0.1 \mu \varepsilon$ with a dynamic range of $80 \mathrm{~dB}$ over a $10 \mathrm{KHz}$ bandwidth. 
Table 2 Design values of the buckled beam strain sensor

\begin{tabular}{|c|l|c|}
\hline$L_{g}$ & Gauge Length & $1000 \mathrm{um}$ \\
\hline$L_{b}$ & Buckled Beam Length & $300 \mathrm{um}$ \\
\hline$\alpha$ & Buckling angle & $5.7^{\circ}$ \\
\hline$N$ & $\begin{array}{l}\text { Number of Fingers on each sensing } \\
\text { beam }\end{array}$ & 37 \\
\hline$g$ & Gap between fingers & $3.6 \mathrm{um}$ \\
\hline$A_{\text {mech }}$ & Mechanical amplification gain & 10 \\
\hline
\end{tabular}

Table 3. Device mechanical parameters

\begin{tabular}{|c|l|c|}
\hline$\omega_{o}$ & $\begin{array}{l}\text { Resonant frequency } \\
\text { (simulated) }\end{array}$ & $8.8 \times 10^{5} \mathrm{rad} / \mathrm{s}$ \\
\hline$m$ & Mass & $0.55 \mu \mathrm{g}$ \\
\hline$Q$ & Quality factor & $\sim 1$ \\
\hline$B W$ & Bandwidth & $10 \mathrm{kHz}$ \\
\hline
\end{tabular}

\section{FABRICATION}

A single mask fabrication process, as shown in Figure 5, was designed to fabricate the device. The process started with a SOI wafer with device/oxide layer of $20 \mu \mathrm{m} / 2 \mu \mathrm{m}$ and device layer resistivity of $0.5-10 \mathrm{ohm}-\mathrm{cm}$. The wafer was first patterned and etched using a DRIE process. Then the wafer was diced, cleaned, and followed by a timed HF release process. Finally, a $200 \AA$ aluminum layer was sputtered on the device to reduce the thermal noise contributed by the device series resistance.

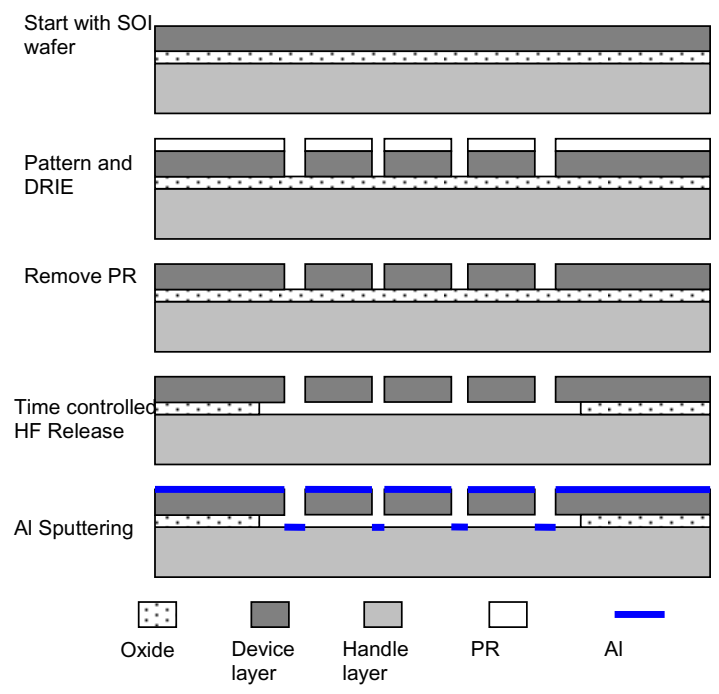

Figure 5. Major fabrication steps.

The SEM picture in Figure 6 shows a fabricated strain sensor with four sets of individual sensors. The size of the sensor (not including the anchors or bonding pads) is around $1000 \mu \mathrm{m}$ by 700 $\mu \mathrm{m}$ with dimensions listed in Table 2 . The selection of buckling angle, $\alpha$, is a trade-off between sensitivity and linearity. The first generation devices were designed for concept verification purpose and the devices were anchored on a silicon substrate and then tested by a three point bending fixture, as will be illustrated in the following section.

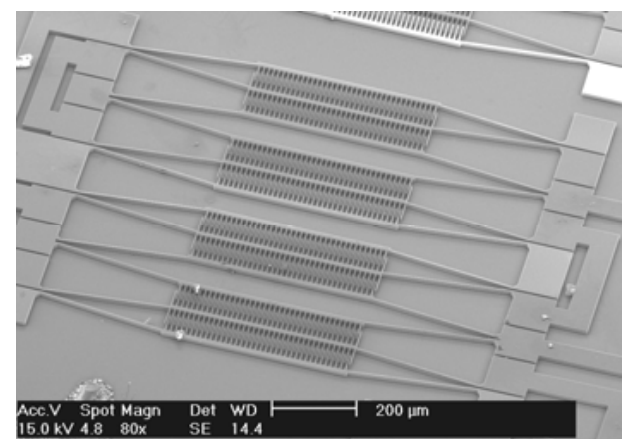

Figure 6. An SEM picture of a buckled beam capacitive strain sensor.

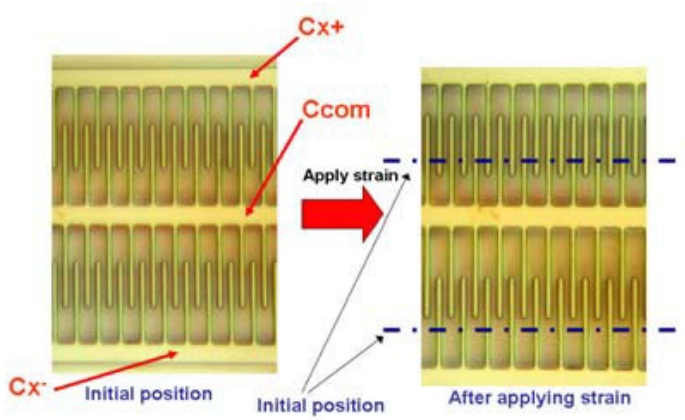

Figure 7. Microphotograph of fingers under deflection

Figure 7 demonstrates an example of the differential capacitance change when a strain is applied. The left figure in Figure 7 shows the initial finger positions when no strain is applied. The initial finger overlapping length for $\mathrm{Cx}^{+}$and $\mathrm{Cx}^{-}$are the same. The right figure shows the finger position changes when a strain is applied, $\mathrm{Cx}^{+}$increase as $\mathrm{Cx}^{-}$decreases, indicating a differential capacitive output.

\section{EXPERIMENTAL RESULTS}

The strain sensor was tested by bending its substrate on a three-point bending test fixture, as shown in Figure 8. The test strip is $30 \mathrm{~mm}$ long with one end clamped and the other end bent by a micrometer. The strain introduced by bending can is calculated by,

$$
\varepsilon_{3-p t}=\frac{3 t\left(L-x_{\text {sensor }}\right)}{2 L^{3}} \Delta Z,
$$

where $\varepsilon_{3-p t}$ is the strain introduced by bending; $t$ is the sensor substrate thickness; $L$ is the length of the substrate; $x_{\text {sensor }}$ is the distance from sensor center to the clamped edge; $\Delta \mathrm{Z}$ is the displacement applied . By manipulating the micrometer, a strain can be applied on the sensor and the capacitance change can be read out by a commercial available $\mathrm{C}-\mathrm{V}$ converter chip. Limited by the micrometer, the test setup can achieve a resolution around $1.5 \mu \varepsilon$.

Figure 9 shows a typical measured sensor capacitance change characteristics as a function of the applied strain. The measured sensitivity of the sensor is $282 \mathrm{aF} / \mu \varepsilon$, which closely matches the hand calculation result $(289 \mathrm{aF} / \mu \varepsilon)$ and FEA result (284 $\mathrm{aF} / \mu \varepsilon)$, indicating an agreement between modeling and measurement.

The measured non-linearity of the sensor is around $1.5 \% \mathrm{FS}$ for tensile strain, which agrees with the simulation results 
$(1.49 \% \mathrm{FS})$ given in Section MODELING B. Sensor Modeling. This non-linearity is introduced by the fact that the mechanical gain changes with the applied strain as the buckling angle changes. It can be improved by optimizing the structural parameters such as buckling angle, shape of the buckling beam, etc.
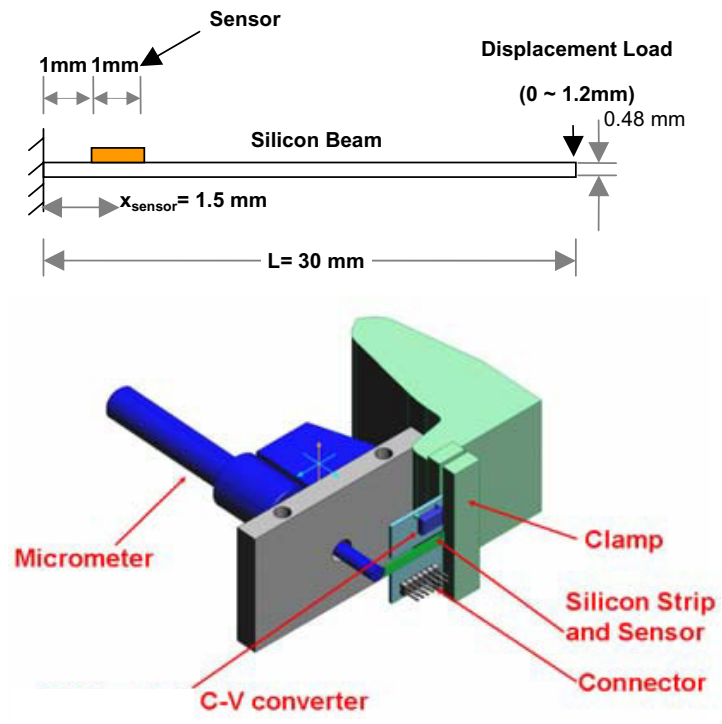

Figure 8. Three point test fixture

The test results also show that the maximum hysteresis is less than $1.5 \mu \varepsilon$. Since the resolution of the testing setup is also around $1.5 \mu \varepsilon$, indicating an improved test fixture is needed to obtain an accurate sensor hysteresis characteristic.

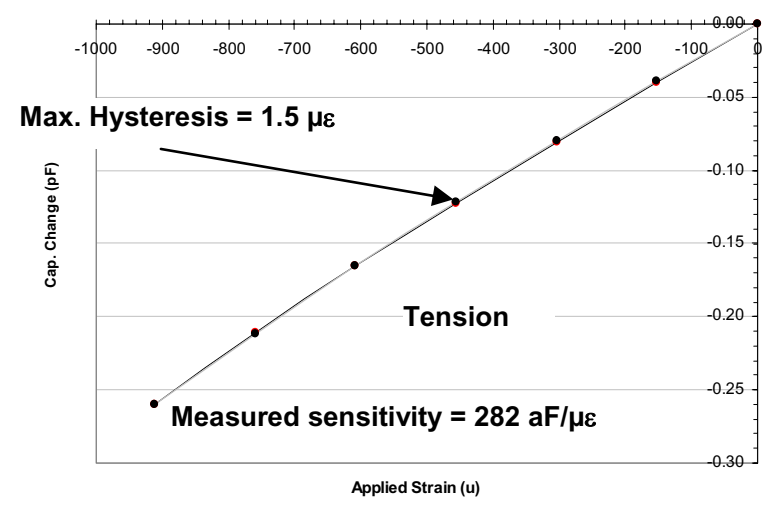

Figure 9. Capacitance change as a function of applied tensile strain.

\section{CONCLUSIONS AND FUTURE WORK}

An innovative capacitive strain sensor employing a mechanical amplification scheme based on the bending of a buckled beam has been presented. The fabricated prototype device achieved a sensitivity of $282 \mathrm{aF} / \mu \varepsilon$, which matches the results obtained analytically and by FEA. The fundamental resolution limit has been studied based on the analysis of the mechanical thermal noise, indicating a sensitivity of $0.1 \mu \varepsilon$ with a dynamic range of $80 \mathrm{~dB}$ over a $10 \mathrm{KHz}$ bandwidth can be expected with a matching interface circuit.
The future work includes the optimization of the structure to achieve an improved sensitivity, linearity, and the device testing fixture, as well as the development of a packaging scheme which integrates the sensor, read out IC and wire connection.

\section{ACKNOWLEDGMENT}

This work was funded by U.S. Army Research Office (ARO) contract \# DAAD 19-02-1-0198. Travel support has been generously provided by the Transducers Research Foundation and by the DARPA MEMS and DARPA BioFlips programs.

\section{REFERENCES}

[1] M. Nagy, C. Apanius, and J. W. Siekkinen, H. V. Estrada, "A user-friendly, high-sensitivity strain gauge”, Sensors, June 2001.

[2] Yogesh B. Gianchandani and Khalil Najafi, "Bent-beam strain sensors", JMEMS, VOL. 5, No. 1, p52-58, March 1996

[3] Liwei Lin, Albert P. Pisano and Roger T. Howe, "A micro strain gauge with mechanical amplifier", JMEMS, Vol. 6, No.4, p313-321, December 1997

[4] Thomas B. Gabrielson, "Mechanical-thermal noise in micromechanical acoustic and vibration sensors", IEEE Transaction, ED Vo1. 40, No. 5, 1993

[5] S. P. Timoshenko and S. woiniwsky-Krieger, "Theory of Plates and shells", 2nd edition, McGraw-Hill, 1970 